\title{
Nature of the solar dynamo at small scales
}

\author{
J. O. Stenflo ${ }^{1,2}$ \\ ${ }^{1}$ Institute of Astronomy, ETH Zurich, CH-8093 Zurich, Switzerland \\ ${ }^{2}$ Istituto Ricerche Solari Locarno, Via Patocchi, CH-6605 Locarno Monti, Switzerland \\ email: stenflo@astro.phys.ethz.ch
}

\begin{abstract}
It is often claimed that there is not only one, but two different types of solar dynamos: the one that is responsible for the appearance of sunspots and the 11-yr cycle, frequently referred to as the "global dynamo", and a statistically time-invariant dynamo, generally referred to as the "local dynamo", which is supposed to be responsible for the ubiquitous magnetic structuring observed at small scales. Here we examine the relative contributions of these two qualitatively different dynamos to the small-scale magnetic flux, with the following conclusion: The local dynamo does not play a significant role at any of the spatially resolved scales, nearly all the small-scale flux, including the flux revealed by Hinode, is supplied by the global dynamo. This conclusion is reached by careful determination of the Sun's noise-corrected basal magnetic flux density while making use of a flux cancellation function determined from Hinode data. The only allowed range where there may be substantial or even dominating contributions from a local dynamo seems to be the scales below about $10 \mathrm{~km}$, as suggested by observations of the Hanle depolarization effect in atomic spectral lines. To determine the fraction of the Hanle depolarization that may be due to the action of a local dynamo, a synoptic program is being initiated at IRSOL (Istituto Ricerche Solari Locarno).
\end{abstract}

Keywords. Sun: atmosphere, magnetic fields, polarization, dynamo

\section{Introduction}

The Sun's dynamo that is responsible for all solar activity with its 11-yr cycle depends on all scales for its operation. It is the statistical left-right symmetry breaking of the convection at all scales by the Coriolis force induced by the Sun's rotation that causes the global, migrating magnetic patterns that characterize the 11-yr cycle. Convective turbulence, a fundamental ingredient of the dynamo, breaks up the generated large-scale structures such that they cascade down the scale spectrum to the magnetic diffusion limit, at scales of $10-100 \mathrm{~m}$, where the field lines cease to be frozen-in and decouple from the plasma. As the symmetry breaking that generates the global pattern takes place and contributes throughout the entire scale range, it is not meaningful to conceptually divide this dynamo into a large-scale and a small-scale part. All scales are interconnected.

From theory and numerical simulations it has however been suggested that, besides the dynamo that is responsible for all of solar activity, there is a second, qualitatively different dynamo that also operates on the Sun and is responsible for the statistically time-invariant small scale structuring of the magnetic field (Petrovay \& Szakaly 1993; Cattaneo 1999; Vögler \& Schüssler 2007).

The dynamo that is responsible for solar and stellar activity is generally referred to as the "global dynamo", to distinguish it from the time invariant, quiet-sun dynamo, which is generally referred to as the "local" or "surface" dynamo. In the present paper we will determine upper, empirical limits to the flux contributions from a local dynamo and thereby show that it is the global dynamo, not the local one, which dominates the observed spatial structuring, even at the smallest of all the resolved scales. 


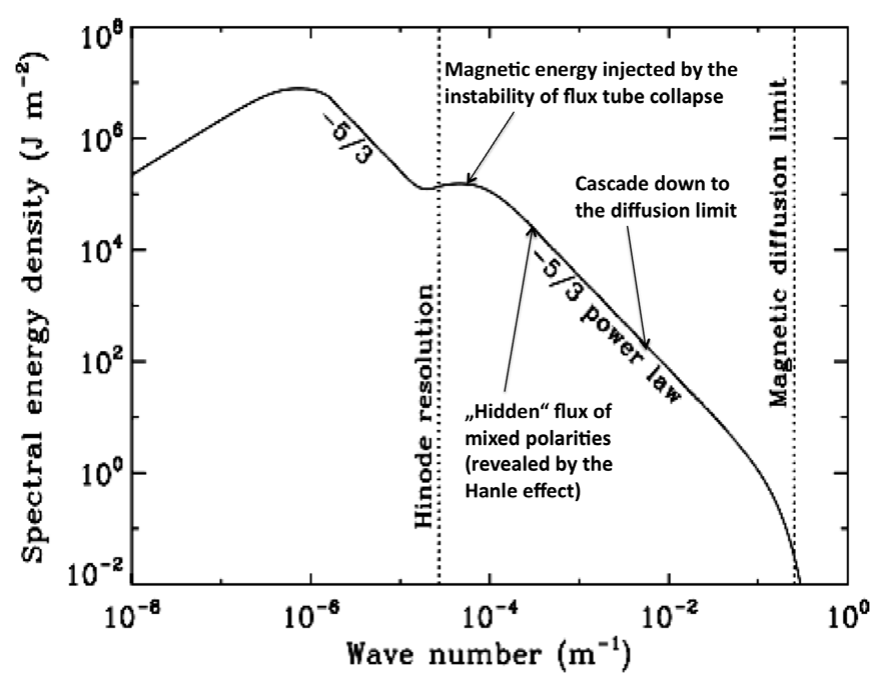

Figure 1. Magnetic energy spectrum of the quiet Sun over a scale range of more than 7 orders of magnitude (adapted from Stenflo 2012a).

These upper limits represent the "basal" magnetic flux density, the minimum average unsigned flux density that can be found in the most quiet regions on the Sun. As however these low average flux densities are completely swamped by measurement noise in the MDI and HMI magnetograms, we have to develop an accurate procedure to determine the noise and remove its influence on the average unsigned flux densities. Fortunately the circumstance that the noise and the intrinsic solar flux densities obey entirely different scaling laws enables us to solve this problem in a well-defined way. For high precision this also requires the use of the empirical, noise-corrected PDF (probability density function) for the flux densities. Both the magnetic field scaling law and the PDF have previously been determined from analysis of Hinode quiet-sun data (Stenflo 2010, 2011). The empirical scaling law is valid over a range that includes the Hinode, HMI, and MDI scales, thus allowing the average unsigned flux densities to be translated across this entire range without the need for any extrapolation.

\section{The Sun's magnetic scale spectrum}

Figure 1 gives an overview of the magnetic energy spectrum for the quiet Sun, spanning more than 7 orders of magnitude. For the spatially resolved scales the spectrum has been determined from direct analysis of Hinode quiet-sun data, while its continuation into the unresolved domain has been inferred by indirect empirical techniques. In particular the bump in the spectrum just beyond the Hinode resolution limit has been inferred from analysis of Hinode line-ratio data that are interpreted in terms of a collapsed, kGtype flux tube population in the $10-100 \mathrm{~km}$ range, and by modeling the influence of this population on the magnetic energy spectrum. The vast amount of "hidden" flux that has been inferred from analysis of the observed Hanle depolarization effect in atomic lines is expected to have its main contribution from the scales just below the range where the flux tubes are believed to reside.

It is a consequence of the turbulent nature of the solar plasma that the magnetic energy spectrum spans all these scales in a continuous manner. Large-scale structures are spontaneously broken up into smaller structures in a cascade down to the end of the scale spectrum. The all-pervasive Coriolis force breaks the left-right symmetry to 
generate a non-zero average helicity at all scales, which is the key process of the cyclic dynamo.

The circumstance that there is substantial magnetic structuring at very small scales is by itself no evidence for the existence of a local dynamo. Only the part of this structuring that is time-invariant and decoupled from the 11-yr cycle could in principle have its origin in a local dynamo.

\section{Scaling laws and noise removal}

Analysis of Hinode magnetic-field data for the quiet Sun has shown that the average unsigned vertical flux density $B_{\text {ave }}$ obeys a scaling law described by the cancellation function

$$
B_{\text {ave }} \sim d^{-\kappa}
$$

(Pietarila Graham et al. 2009; Stenflo 2011), where $d$ is the scale size (side of the squareshaped smoothing window), and $\kappa$ is the cancellation exponent. By numerical smoothing of the Hinode data to simulate the effect of different spatial resolutions, a cancellation function with a small, approximately constant value of $\kappa$ across the whole range from the Hinode 0.3 arcsec scale up and beyond the MDI 4 arcsec scale is found. According to the analysis of Pietarila Graham et al. (2009) the numerical value of $\kappa$ is 0.26 , while according to Stenflo (2011) it is 0.13 . Both these values are much smaller than $\kappa$ for random noise, which is unity as a consequence of Poisson statistics. Due to the great difference in $\kappa$ it becomes possible to achieve a clean separation between the noise and the intrinsic solar contribution.

As the size of the smoothing window is increased, the observed, noise-affected apparent flux density $B_{\text {app }}$ initially decreases steeply due to the steep scaling law of the noise component, but subsequently levels out to asymptotically approach the much shallower slope of the solar scaling law. This behavior is illustrated in Fig. 2 for $B_{\text {app }}$ (solid lines) as determined from the disk-averaged vertical flux density in two different MDI magnetograms, one recorded with 5 min integration, the other with 1 min integration. The dotted curves represent the intrinsic solar components governed by a scaling law with cancellation exponent 0.13 .

To accurately combine the solar and noise contributions we need to know the empirical shape of the intrinsic (noise-free) PDF (probability density function) for the vertical flux densities. This PDF has previously been determined from analysis of Hinode quietsun data and found to be characterized by an extremely narrow core peak, represented by a stretched exponential centered at zero flux density, and quadratically declining "damping" wings.

The effect of noise is to convolve the solar PDF with the Gaussian PDF of random noise. Through numerical Gaussian convolution of the solar PDF, we have found that the relation between the apparent, noise-affected average flux density $B_{\text {app }}$ and its noise-free counterpart $B_{\text {ave }}$ can be analytically represented by

$$
\begin{aligned}
B_{\text {ave }} & =\left[B_{\text {app }}^{\alpha}-(0.798 \sigma)^{\alpha}\right]^{(1 / \alpha)}, \\
\alpha & =1.36-0.004 \sigma+0.0034 B_{\mathrm{app}}
\end{aligned}
$$

over the relevant parameter range. $\sigma$ is the standard deviation of the Gaussian noise distribution. The reason for the factor 0.798 is that the average unsigned $x$ value of a Gaussian distribution with standard deviation $\sigma$ is $0.798 \sigma$. A practically identical relation is obtained if we replace our solar PDF with a Lorentzian function, which shows that the detailed shape of the solar PDF core region is unimportant in this context. 


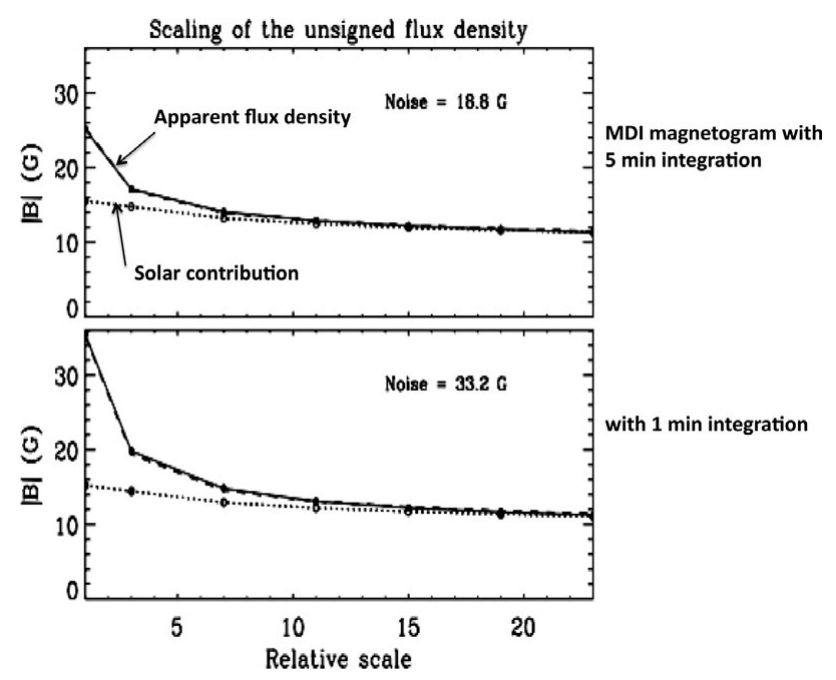

Figure 2. Illustration of the procedure for noise removal from the observed (apparent) average vertical flux densities, for the example of two MDI magnetograms obtained with different integration times. The relative scale of the horizontal axis is given in pixel units. With a model that uses the greatly different scaling laws for the noise and the solar component, the noise-free average flux density (dotted lines) can be determined from its noise-affected counterpart (solid curves). With the fitted values for the noise $\sigma$ of 18.8 and $33.2 \mathrm{G}$, respectively, the model (dashed curves) reproduces the observed curves nearly perfectly.

Applying this noise model to all the 73,838 SOHO/MDI 96 min cadence magnetograms, we obtain excellent model fits to the observed average unsigned flux density, as illustrated in Fig. 2 by the nearly perfect agreement between the solid (observations) and dashed (model) curves. The model has two free parameters to be determined by the fit: the noise $\sigma$, and the value of the noise-free $B_{\text {ave }}$ in the unsmoothed magnetogram. With a fixed value of 0.13 for the cancellation exponent $\kappa$ that governs the $B_{\text {ave }}$ scaling (while for random noise, $\kappa$ is always $=1$ ), the same values of $\sigma$ are found from the model fit for all the magnetograms that have the same integration time (which is either 1 or $5 \mathrm{~min}$ ), regardless of the value of $B_{\text {ave }}$, which varies greatly with the phase of the solar cycle. With other choices for $\kappa$ the value of $\sigma$ becomes dependent on $B_{\text {ave }}$, which is unphysical, since $\sigma$ should represent measurement noise that is independent of the conditions on the Sun. It is gratifying that the value of $\kappa$ that is so demanded by physical consistency is the same as previously determined by an entirely independent type of analysis of Hinode quiet-sun data (Stenflo 2011). This consistency is further confirmation of the validity of this scaling law over the range that spans the Hinode and MDI scales.

A more detailed account of the noise removal procedure and its application to the MDI and HMI data for the determination of the basal flux density can be found in Stenflo (2012b).

\section{Basal flux density from MDI analysis}

For each of the MDI magnetograms that span the 15-yr period May 1996 - April 2011, we have converted the directly measured line-of-sight component of the magnetic flux density to vertical flux density through division by $\mu$, the cosine of the heliocentric angle, assuming that on average the field is oriented in the vertical direction due to the strong buoyancy forces acting on the $\mathrm{kG}$ type flux tubes, which carry most of the flux in the photosphere. We then average these vertical flux densities over all the pixels, but 


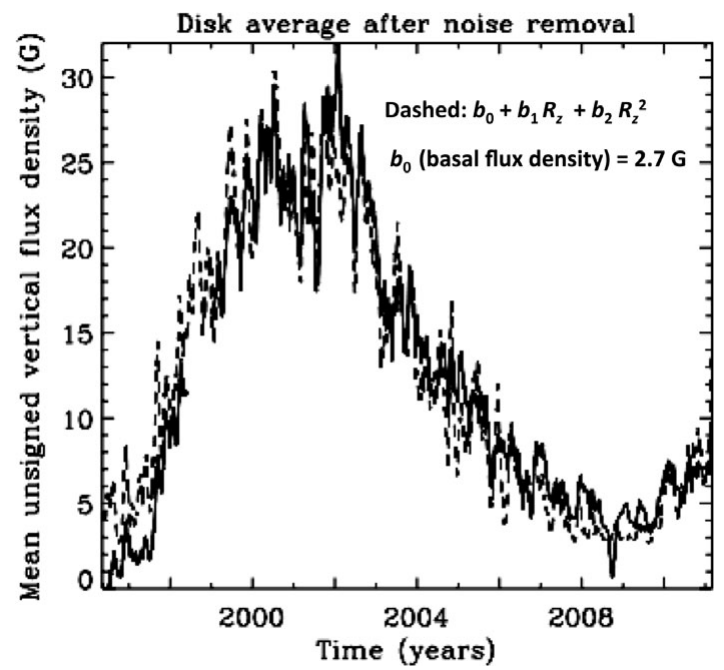

Figure 3. Noise-free, disk averaged unsigned vertical flux density $B_{\text {ave }}$ (solid curve), compared with a second-order polynomial of the sunspot number (dashed curve). The nearly perfect correlation allows us to interpret the constant polynomial coefficient $b_{0}$ as the basal vertical flux density, representing its value in the absence of solar activity and sunspots.

exclude a limb zone with a width of $10 \%$ of the disk radius. This average defines our noise-affected value of $B_{\text {app }}$. It is then converted to its noise-free counterpart $B_{\text {ave }}$ with our noise removal model.

Figure 3 shows as the solid line the so determined values of $B_{\text {ave }}$ as a function of time for the 5-min integration MDI magnetograms for the whole period covered by the data set (excluding a data gap during 1998.4 - 1999.0). The corresponding results for the 1min MDI magnetograms are not illustrated here, because they look the same except for their larger noise fluctuations. Since the solid curve has a striking resemblance to the corresponding curve for the sunspot number $R_{z}$, we have tested the sunspot correlation by fitting $B_{\text {ave }}$ to a second-order polynomial $b_{0}+b_{1} R_{z}+b_{2} R_{z}^{2}$ in the sunspot number. This test reveals a nearly one-to-one correspondence between $B_{\text {ave }}$ and the sunspot number, as shown by the dashed model curve in Fig. 3 .

Since $B_{\text {ave }}$ can be modeled so closely by the sunspot number, we may interpret the constant, cycle-independent term $b_{0}$ of the polynomial fit as the value of $B_{\text {ave }}$ in the absence of sunspots, in other words, as the basal unsigned flux density. This basal value is found to be $2.7 \mathrm{G}$ for the set of 5 -min integration MDI magnetograms.

\section{Basal flux density from HMI analysis}

The noise-removal model that we used for the MDI magnetograms applies equally well to the SDO/HMI data. As the HMI data set so far mainly covers the deep minimum phase of the solar cycle and a correlation with sunspots therefore cannot be done, it is not meaningful to do averaging over the whole solar disk. Instead we have for HMI defined the noise-affected average unsigned flux density $B_{\text {app }}$ as the average over the innermost $10 \%$ of the radius $r_{\odot}$, i.e., $r / r_{\odot}<0.1$. Since $\mu \approx 1.0$ over this whole region, the lineof-sight component of the flux density equals the vertical component, no conversion is needed.

When applying our noise model to the HMI disk center data with cancellation exponent $\kappa=0.13$, we find a value of the noise $\sigma(8.0 \mathrm{G})$ that is independent of $B_{\text {ave }}$, as required by 


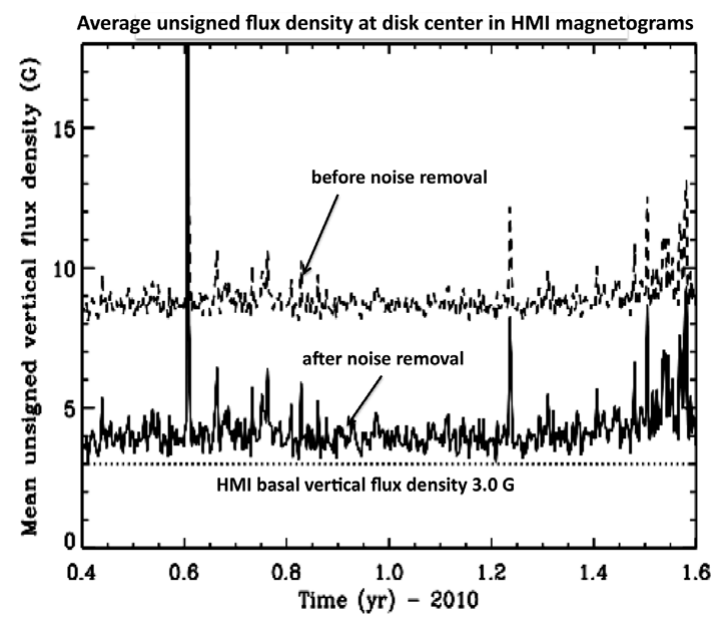

Figure 4. Time series of the noise-affected $B_{\text {app }}$ (dashed line), defined as the average unsigned vertical flux density within the disk center region $r / r_{\odot}<0.1$ of the HMI magnetograms, here selected with a cadence of $24 \mathrm{hr}$. When noise is removed $B_{\text {app }}$ becomes $B_{\text {ave }}$, shown as the solid line. Its lower envelope is $3.0 \mathrm{G}$, which represents the basal flux density for the HMI spatial scale.

physical consistency. Other choices of $\kappa$ do not have this property, which again supports the validity of this scaling law.

In Fig. 4 the directly observed, noise-affected $B_{\text {app }}$ is given as a function of time for the period 2010.4 - 2011.6 as the dashed curve, while its noise-corrected counterpart $B_{\text {ave }}$ is the solid curve. Their spiky appearance is not due to noise but is solar, due to the circumstance that we have only selected one HMI magnetogram per $24 \mathrm{hr}$. Since solar rotation brings significantly different magnetic features into the analysed disk center region, $B_{\text {app }}$ becomes jumpy when using such course time cadence. These solar spikes get mapped one by one when converting the dashed curve into the solid one, the conversion procedure does not create additional fluctuations.

The basal vertical flux density can now be obtained simply as the lower envelope to the solid curve, $3.0 \mathrm{G}$, below which the average flux density never dips, even in the most quiet solar regions during this record deep cycle minimum phase.

Using our cancellation function with $\kappa=0.13$, we can translate the HMI basal flux density to its corresponding value at the MDI scale. Since the spatial resolutions of HMI and MDI differ by a factor of 4 , the $3.0 \mathrm{G}$ HMI value translates to $3.0 \times 4^{-0.13}=2.5 \mathrm{G}$, which is in nearly perfect agreement with the $2.7 \mathrm{G}$ value that was based on the correlation of the 5-min integration MDI magnetograms with the sunspot number.

Similarly we can translate the HMI basal flux density from the 1 arcsec HMI scale to the Hinode 0.3 arcsec scale, since our cancellation function was derived from Hinode data and later validated by the HMI and MDI data. This gives $3.0 \times 0.3^{-0.13}=3.5 \mathrm{G}$, a small number compared with the average unsigned flux densities of typically $10-15 \mathrm{G}$ found from Hinode analysis of quiet regions (Lites et al. 2008; Stenflo 2011), indicating that the quiet regions that were analysed with Hinode were not representative of the basal flux level but contained substantially more flux. This issue will be examined in the next section.

\section{Variations of quiet-sun magnetic fields}

During the period covered by our HMI analysis there were no active regions near disk center, the fluctuations that we see from day to day in Fig. 4 represent quiet regions with 


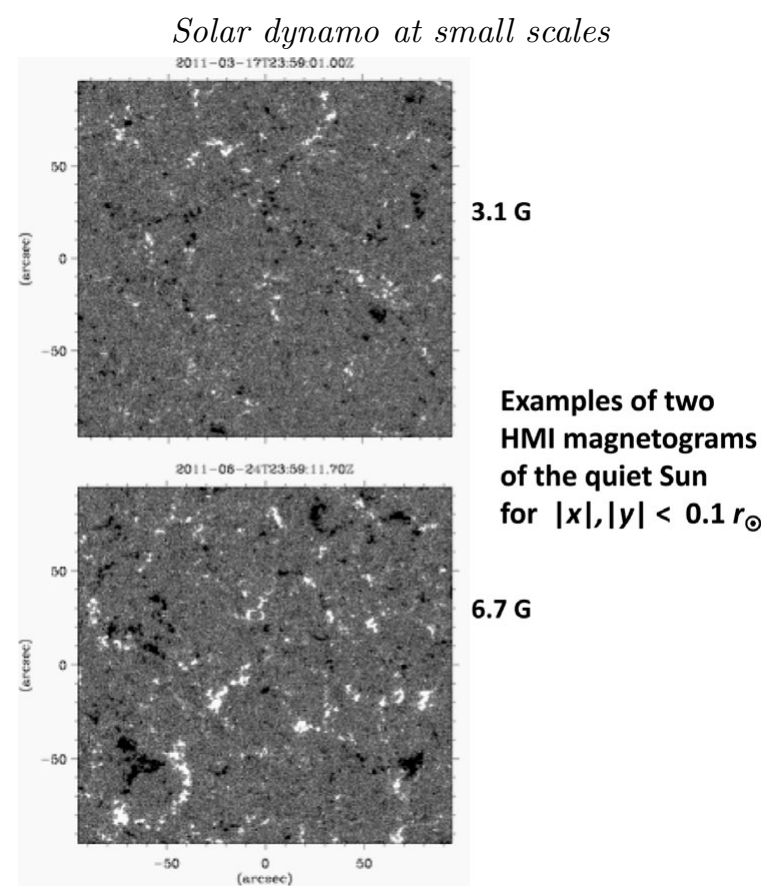

Figure 5. Comparison between the quiet-sun disk-center regions of two HMI magnetograms, obtained at times 2011.2086 (upper panel) and 2011.4796 (lower panel), representing regions with basal flux density and with 2.2 times the basal flux density, respectively. The grey-scale cuts $( \pm 50 \mathrm{G})$ are the same for the two panels.

varying average flux densities. Such variations in the amount of flux can only come from the global dynamo, since the local dynamo has nothing to do with the solar cycle and therefore is time invariant.

The circumstance that there is a non-zero basal flux density in the absence of any sunspot activity does not imply that the local dynamo must be the source of this basal flux. Instead it is to be expected that the large amounts of flux that have been generated by the global dynamo during the course of the 11-yr cycle do not instantly disappear when the solar activity becomes zero, but that flux remnants will linger around for extended periods of time and only slowly decay asymptotically towards zero if there is no supply of new flux. For this reason the determined non-zero basal flux density only defines an upper limit to the contributions from a hypothetical local dynamo, but not evidence for its existence.

The validity of the conclusion that the noise-corrected HMI basal flux density is approximately $3 \mathrm{G}$, and not significantly less (like zero) or larger, can easily be verified by direct visual inspection of the corresponding magnetograms and by identifying the parts of the PDF that contribute to the bulk of the observed flux. For this purpose we have selected the disk-center magnetograms from two days, one representing the basal, minimum average flux density (upper panel of Fig. 5, recorded at time 2011.2086), the other having an average flux density more than twice as large $(6.7 \mathrm{G}$, lower panel of Fig. 5, recorded at time 2011.4796). While it is obvious that there is much more flux in the quiet region of the lower panel, it is striking that the qualitative appearance of the patterns is very similar in the two panels. Thus the basal flux region exhibits the same kind of strongly intermittent pattern that is network-like on the supergranulation scale.

Before noise correction, the average unsigned flux densities of the two regions were 8.2 and $10.8 \mathrm{G}$, thus in proportion $1: 1.3$, which is a small difference in comparison with the 


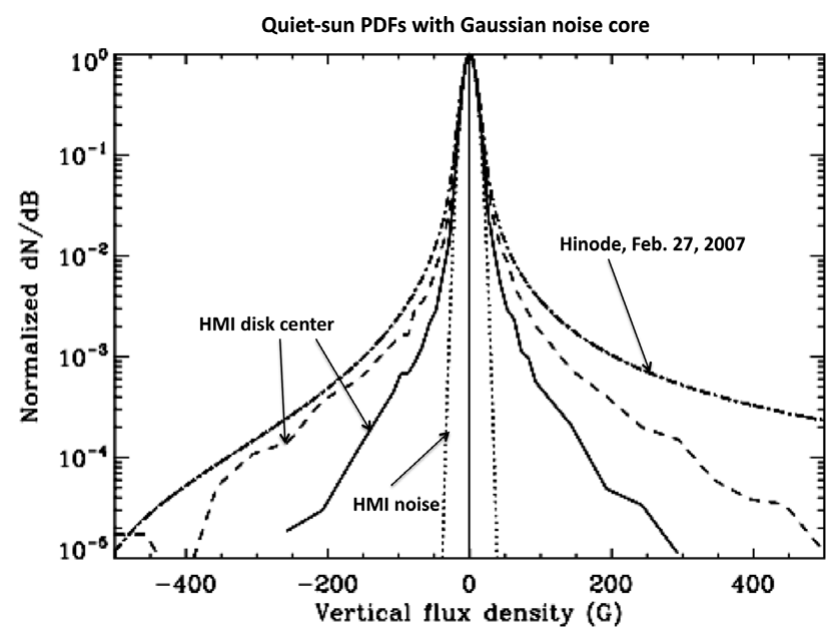

Figure 6. Amplitude-normalized probability density functions (PDF) for the upper and lower magnetogram of Fig. 5 (solid and dashed curve, respectively), for the HMI noise distribution (dotted curve), and for the PDF that has been determined from Hinode observations of February 27, 2007 (Stenflo 2010), after noise removal and subsequent convolution with the HMI noise Gaussian, to allow comparison of the different PDF wings on the same scale. While the solid curve is representative of the basal flux density, everything above it expresses the cycle dependence of the background quiet-sun flux.

proportion $3.1: 6.7=1: 2.2$ after noise correction. If the basal flux density were much closer to zero than $3 \mathrm{G}$, then the above proportion would escalate, and we would not see as much magnetic structures in the upper panel as we do see. This visual check confirms that our non-zero basal flux density cannot be very different from our determined value.

To further clarify the role of noise and the entirely different nature of the intrinsic solar magnetic pattern we show in Fig. 6 the PDFs of the two magnetograms of Fig. 5, as the solid line for the basal magnetogram, and as the dashed line for the magnetogram with 2.2 times the basal flux density. These PDFs are uncorrected for noise, which means that they are smeared by convolution with the $\sigma=8.0 \mathrm{G}$ Gaussian noise distribution, which is plotted separately as the dotted line. The circumstance that this Gaussian is practically indistinguishable from the shapes of the core regions of the solid and dashed PDFs implies that the width of the intrinsic (noise-free) PDF core peak is much smaller than the $8 \mathrm{G}$ of the noise Gaussian.

The much slower, approximately quadratical decline of the PDF wings implies that they are nearly unaffected by the noise convolution and therefore represent the wing shape of the intrinsic PDFs. Practically all the noise-corrected average unsigned flux density comes from the PDF wings, the contribution from the core region is insignificant due to the extremely narrow intrinsic width of the core peak. Before noise correction, however, the average unsigned flux density is dominated by the contribution from the core region, where all the noise resides.

The extended PDF wings are a signature of spatial intermittency, since the high flux densities in the wings combined with the low occurrence probability implies that the corresponding flux elements are relatively rare, and that they must therefore be statistically well separated (i.e., intermittent). Since the bulk of the flux comes from the PDF wings, this implies that most of the flux is highly intermittent, which confirms the visual impression that we get from the magnetograms in Fig. 5. As all the noise in the plotted magnetograms has values far below the chosen grey-scale cuts of $\pm 50 \mathrm{G}$, the noise is 
inconspicuous. The visual impression of the pattern comes exclusively from the contributions of the PDF wings. Still it is the inconspicuous noise background that dominates the apparent, average unsigned flux density $B_{\text {app }}$.

For comparison we show as the dash-dotted line in Fig. 6 the analytical representation of the noise-free PDF derived from Hinode SOT/SP observations of the quiet-sun disk center on February 27, 2007 (Stenflo 2010). However, before plotting we have convolved it with the $\sigma=8.0 \mathrm{G}$ HMI Gaussian noise distribution, because only when all the PDFs in the figure have been smeared with the same noise function and been amplitude normalized, it becomes possible to compare the levels of the different PDF wings with each other on a common scale. We see that the Hinode PDF wings are substantially more elevated than the PDF wings of our two HMI magnetograms and therefore contribute much more flux. The main reason why analysis of Hinode quiet-sun data has indicated much higher flux densities than what one can expect from our small basal flux density is simply because the observations were not carried out during the deepest part of the solar activity minimum. Therefore there was much more background magnetic flux on the quiet Sun. Due to the shallowness of the cancellation function the elevated Hinode PDF wings cannot be explained in terms of scaling between the 1 and 0.3 arcsec scales.

For this reason we can safely say that the bulk of the quiet-sun vertical magnetic flux observed with Hinode cannot have its origin in a local dynamo, since the Hinode flux densities much exceed the basal flux density.

\section{Basal flux density of the horizontal fields}

The Hinode SOT/SP instrument has the capability of measuring all Stokes parameters and therefore in principle of determining the magnetic field vector. The polarimetric precision is high: for the circular polarization (Stokes $V$ ) it is $0.047 \%$ (in units of the continuum intensity), for the linear polarization it is even better, $0.035 \%$ in each of Stokes $Q$ and $U$ (Stenflo 2011). For those who are not so familiar with the profound difference between the longitudinal and the transverse Zeeman effects, the high polarimetric sensitivity for the linear polarization may give the completely misleading impression that transverse and longitudinal magnetic fields can be determined with comparable accuracy.

The approximate conversion laws between the Stokes $V, Q$, and $U$ amplitudes (in units of the continuum intensity) and the longitudinal and transverse flux densities $B_{\|}$and $B_{\perp}$ for field strengths below about $0.5 \mathrm{kG}$, assuming for simplicity that the field is resolved (filling factor unity), are

$$
\begin{aligned}
B_{\|}(\mathrm{G}) & =29.4 V(\%), \\
B_{\perp}(\mathrm{G}) & =184\left[Q(\%)^{2}+U(\%)^{2}\right]^{1 / 4},
\end{aligned}
$$

where the proportionality factors have been determined by radiative-transfer modeling of the Fe I $6301 \AA$ line used by Hinode (Stenflo 2011). We note that while the relation between field strength and polarization is linear in the longitudinal case, it is highly non-linear with a large proportionality factor in the transverse case.

With these equations we can use Monte Carlo techniques to convert the Gaussian noise distributions in Stokes $V$ (with $\sigma=0.047 \%$ ) and in $Q$ and $U$ (each with $\sigma=0.035 \%$ ) into PDFs for the apparent magnetic flux density. The result is shown in Fig. 7 (same as was plotted on a linear scale in Fig. A.1 of Stenflo 2011). If these PDFs would mistakenly be assumed to represent solar properties, they would suggest that the Sun is completely swamped by horizontal magnetic flux with typical flux densities of order $40 \mathrm{G}$, with a 


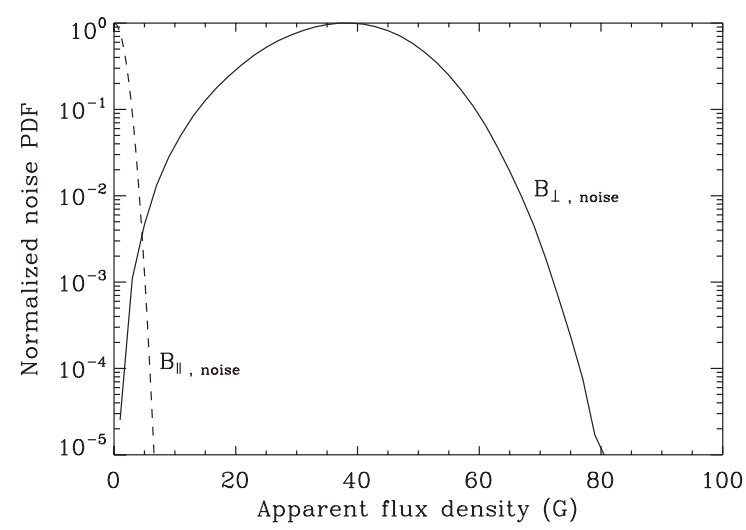

Figure 7. PDFs of the longitudinal and transverse apparent flux densities, exclusively due to Gaussian polarimetric noise in the Hinode SOT/SP recordings of the Fe I $6301 \AA$ line. As the noise PDF for the horizontal flux density is ubiquitous and time invariant, it masquerades as a basal flux density.

wide distribution of field strengths. In comparison, the contribution of the vertical fields would seem insignificant.

The PDF for the directly determined apparent flux densities, which is composed of a combination of the noise and solar contributions, will always be extended to larger flux densities than the noise PDFs alone. Thus the distributions in Fig. 7 represent ubiquitous, time-invariant, lower limits to the observed PDFs, since the instrumental noise hardly changes and at least does not improve over the Hinode mission. The noise PDF for $B_{\perp}$ therefore masquerades as a huge basal flux density, although it is nothing of the sort.

If one wants to make a claim from observations that horizontal fields are of significance for the local dynamo, then one must first deal with the formidable problem how to remove the colossal noise contribution from the apparent horizontal flux density. In the previous sections we have addressed the noise removal problem for the line-of-sight flux density, but because of the approximately 25 times larger noise level of the transverse field (after conversion to $\mathrm{G}$ units), and most of all because of its non-linear conversion behavior, the noise problem for the horizontal fields is of a completely different magnitude.

There have been repeated claims that Hinode data have revealed the existence of much more horizontal than vertical flux in the quiet-sun photosphere, or in other words, that the angular distribution of the field vectors is much more pancake like than isotropic. Related to this are also claims that the vast amounts of such horizontal flux must be due to a local dynamo, since it is always present. Unfortunately none of these claims is based on any serious attempt to address the formidable noise removal problem for the transverse fields.

When the noise issue has been seriously addressed in determinations of the angular distribution of the field vectors, it is found that instead of being pancake like, the distribution favors the vertical direction, but becomes increasingly isotropic as we approach the limit of small flux densities (Stenflo 2010). This implies that there is more vertical than horizontal flux on the quiet Sun, and that there is no valid evidence for the existence of a local dynamo from observations of horizontal magnetic fields.

\section{A role for the local dynamo at scales below $10 \mathrm{~km}$}

Our low basal vertical flux density of $3.0 \mathrm{G}$ determined from analysis of HMI data, which corresponds to $3.5 \mathrm{G}$ at the Hinode resolution, implies that the bulk of the 
magnetic structuring seen by Hinode in quiet solar regions has nothing to do with a local dynamo but represents background magnetic flux that has been generated by the global dynamo. The mentioned basal flux densities represent upper limits to the contributions of a hypothetical local dynamo, but not evidence for its existence, since we expect that there will always remain some long-lived left-over non-zero background flux when the solar activity with its supply of new flux from the Sun's interior temporarily disappears. Our low upper limit therefore indicates that the local dynamo does not play any significant role on the Sun at any of the spatially resolved scales, including the Hinode scale, and that the small-scale magnetic flux that we see has instead been produced by the global dynamo.

This does not rule out the possibility that the local dynamo can become significant at smaller, still unresolved scales. The only known way to empirically address this issue is through observations of the Hanle effect, through its depolarizing effect on the spectral-line polarization caused by coherent scattering. The observed depolarization can be converted into a turbulent field strength (Stenflo 1982), which is unobservable with the Zeeman effect due to cancellation of the contributions from unresolved opposite polarities. Such cancellations do not occur for the Hanle effect due to its different symmetry properties.

While the Hanle effect in optically thin molecular lines gives us a turbulent field that is too weak (of order $10 \mathrm{G}$ ) to point towards the significant role of a local dynamo, the Hanle effect in atomic lines, in particular the Sr I $4607 \AA$ line (Trujillo Bueno et al. 2004) and in the optically thick molecular CN lines (Shapiro et al. 2011), indicates much stronger turbulent fields, of order $60 \mathrm{G}$ or more, which is too large to be reached by a scaling law that connects with the resolved flux densities supplied by the global dynamo. This strongly suggests a dominating role for the local dynamo at the scales responsible for the Hanle depolarization signatures.

The apparently contradictory low Hanle field strengths found with the optically thin molecular lines can be given a consistent explanation if most of the strong fields are located in the intergranular lanes, which do not contribute much to the formation of these molecular lines. This explanation however still needs to be verified by future observations of the Hanle effect with high spatial resolution, to allow the relative contributions from the intergranular lanes and the cell interiors to be clearly separated from each other.

Whether or not these strong turbulent fields have anything to do with the local dynamo depends on how much of them are statistically time invariant, independent of the phase of the solar cycle. This remains a controversial issue. On the one hand the general appearance of the Second Solar Spectrum (the linearly polarized spectrum that is exclusively formed by coherent scattering processes) is found to vary significantly with the solar cycle (Stenflo 2003), and such variations can only be magnetically induced (via the Hanle effect), indicating substantial cycle variations of the "hidden" field. On the other hand Trujillo Bueno et al. (2004) have compiled evidence from various observations of the $\mathrm{Sr}$ I $4607 \AA$ line that there is no significant variation of the $60 \mathrm{G}$ type turbulent field with the cycle.

The only known way to quantitatively resolve this issue is through a synoptic program for the Hanle depolarization in atomic lines like the Sr I line. A reason why this has not been done before is that trustworthy synoptic results can only be obtained with a differential method, allowing us to normalize the recorded scattering polarization amplitude to something that is independent of the solar cycle. The problem has been that good Hanle lines like the Sr I $4607 \AA$ line are spectrally isolated with no suitable reference lines close by, and that we cannot maintain the polarization scale or the chosen limb distance 
of the spectrograph slit to be sufficiently constant over time scales of the solar cycle. We have however recently found an elegant solution to this problem, as follows:

The line with the largest scattering polarization amplitude in the whole visible spectrum is the Ca I $4227 \AA$ line. It is only affected by the Hanle effect in its Doppler line core, while its unblended prominent polarization peak in the blue line wing is immune to the Hanle effect and therefore invariant with the solar cycle. While this blue wing peak therefore can serve as an ideal normalization reference for a synoptic program, its spectral location is far from suitable photospheric Hanle lines like the Sr I $4607 \AA$ line. However, when the Sr line is observed in the 11th grating order and the Ca line in the 12 th order, they fall adjacent to each other in the spectral focus. With two square-shaped order-separating interference filters side by side immediately before the CCD detector, the two lines can be recorded simultaneously on the same CCD. This assures that both lines get recorded at the identical limb distance and seeing conditions. The ratio between the Sr polarization amplitude and the Ca blue wing amplitude is then a differential measure of the Hanle depolarization in the Sr line on a scale that is stable over solar cycle time scales. A synoptic program based on this technique will now be started at IRSOL (Istituto Ricerche Solari Locarno) with the ZIMPOL-3 imaging Stokes polarimeter.

The spatial scales responsible for the observed Hanle depolarization signatures must be much smaller than the resolved scales, otherwise much more of these "hidden" fields would already be visible in magnetograms. Based on indirect arguments (Stenflo 2012a) it is conjectured that the main contribution to these Hanle signatures comes from scales that are below the scale range of $10-100 \mathrm{~km}$, where most of the collapsed, kG-type flux tubes are expected to reside (cf. Fig. 1). While the local dynamo appears to be insignificant at all the resolved scales, it could dominate at scales below $10 \mathrm{~km}$. This would raise the small-scale end of the magnetic energy spectrum and make it shallower than the reference spectrum of Fig. 1. Current data from the Hanle effect suggest that this might indeed be the case. With the synoptic program that is being started at IRSOL we hope to determine the fraction of the observed Hanle depolarization signatures that could be attributed to the action of a local dynamo.

\section{References}

Cattaneo, F. 1999, ApJ (Letters), 515, L39

Lites, B. W., Kubo, M., Socas-Navarro, H., et al. 2008, ApJ, 672, 1237

Petrovay, K. \& Szakaly, G. 1993, A\&A, 274, 543

Pietarila Graham, J., Danilovic, S., \& Schüssler, M. 2009, ApJ, 693, 1728

Shapiro, A. I., Fluri, D. M., Berdyugina, S. V., Bianda, M., \& Ramelli, R. 2011, A\& A, 529, A139

Stenflo, J. O. 1982, Solar Phys., 80, 209

Stenflo, J. O. 2003, in: J. Trujillo Bueno \& J. Sánchez Almeida (eds.), Solar Polarization 3, Astron. Soc. Pacific Conf. Series, Vol. 307, 385

Stenflo, J. O. 2010, $A \& A$, 517, A37

Stenflo, J. O. 2011, $A \& \mathcal{E} A, 529$, A42

Stenflo, J. O. 2012a, $A \& A$ A, 541, A17

Stenflo, J. O. 2012b, A\&SA, 547, A93

Trujillo Bueno, J., Shchukina, N., \& Asensio Ramos, A. 2004, Nature, 430, 326

Vögler, A. \& Schüssler, M. 2007, A\&̧A, 465, L43 